\title{
Note on the Geometry of two Binary quadratics in the complex plane.
}

By W. SAdDler.

(Read 14th June 1924. Received 30th June 1924).

If

$$
\begin{gathered}
a z^{2}+2 b z+c=0 \\
a^{\prime} z^{2}+2 b^{\prime} z+c^{\prime}=0
\end{gathered}
$$

represent the points $A, B$ and $C, D$ respectively and if

$$
a c^{\prime}+a^{\prime} c-2 b b^{\prime}=0
$$

then $A, B, C, D$ are four harmonic points on a circle. If $O$ is the mid point of $A B$ then $O A^{2}=O C . O D$ and $\angle C O B=\angle D O B$.*

To construct the Jacobian of these two quadratics, namely

$$
\left(a b^{\prime}-a^{\prime} b\right) z^{2}+\left(a c^{\prime}-a^{\prime} c\right) z+\left(b c^{\prime}-b^{\prime} c\right)=0
$$

we proceed as follows.

The mid point of $C, D$ being $-\frac{b^{\prime}}{a^{\prime}}$ has as its fourth harmonic with respect to (1) the point

$$
z=\frac{b b^{\prime}-c a^{\prime}}{b a^{\prime}-a b^{\prime}}
$$

so the mid point of $A, B$ i.e. $-\frac{b}{a}$ has as its four harmonic with respect to (2) the point $z=\frac{a c^{\prime}-b b^{\prime}}{b a^{\prime}-a b^{\prime}}$.

The mid point of these two points just found will be $\frac{1}{2} \cdot \frac{a c^{\prime}-a^{\prime} c}{b a^{\prime}-a b^{\prime}}$ which is the mid point of the pair of points given by (3). We know that the pairs (1) and (2) are harmonic with respect to (3), and as we can thus determine the mid point of the pair (3) we can actually construct these points. Thus if $M$ is this mid point : let $R M S$ bisect the angle $A M B$. The perpendiculars to $R S$ at $M$ and

* \$ Grace and Young, Algebra of Invariants, pages 209, 210. 
to $A B$ at its mid point will meet at a point $O$ which is the centre of the circle, with $O A$ as radius, which will meet the line $R S$ in the points represented by the Jacobian.

Taking the quadratic giving $A, B$ as $z^{2}+p z+q=0$ and $C, D$ as $z^{2}+p^{\prime} z+q^{\prime}=0$ we know that their Jacobian is harmonic to all points given by $(4) \ldots \lambda\left(z^{2}+p z+q\right)+\mu\left(z^{2}+p^{\prime} z+q^{\prime}\right)=0$, and in the case where $\lambda, \mu$ are real we can determine the mid point of the pair given by (4), and hence actually construct the points in this case.

A special result is the following.

Take any two points $P, Q$ : the foci of the conic which touches the triangle $A B P$ at the mid points of the sides being given by $\frac{d f}{d z}=0$ where

$$
\begin{aligned}
f=\left(a z^{2}+2 b z+c\right)(z+p) & =0 \quad \text { is } \\
3 a z^{2}+2 z(2 b+p a)+(c+2 b p) & =0
\end{aligned}
$$

so for $A, B, Q \quad\left(a z^{2}+2 b z+c\right)(z+q)=0$ is

$$
3 a z^{2}+2 z(2 b+q a)+(c+2 b q)=0 .
$$

Let these points be $F_{1} F_{2} F_{3} F_{4}$. The pair of points Harmonic to both $F_{1} F_{2}$ and $F_{3} F_{4}$ is given by

$$
3 a^{2} z^{3}+6 a b z+\left(4 b^{2}-a c\right)=0
$$

which is independent of $P$ and $Q:$ call these points $M, N$.

Treat the quadratic giving $C, D$ in a similar manner and we get two points $M^{\prime} N^{\prime}$ given by $3 a^{\prime 2} z^{2}+6 a^{\prime} b^{\prime} z+\left(4 b^{\prime 2}-a^{\prime} c^{\prime}\right)=0$. The two points harmonic to $M, N, M^{\prime}, N^{\prime}$ are given by

$$
\begin{aligned}
\dot{4}\left(a b^{\prime}-a^{\prime} b\right) & \left\{a a^{\prime} z^{2}+\left(a b^{\prime}+a^{\prime} b\right) z+a^{\prime} b^{\prime}\right\} \\
& -a a^{\prime}\left\{z^{2}\left(a b^{\prime}-a^{\prime} b\right)+\left(a c^{\prime}-a^{\prime} c\right) z+\left(b c^{\prime}-b^{\prime} c\right)\right\}=0 .
\end{aligned}
$$

The first part is the quadratic giving the mid points of the pair of points $A B, C D$, and the second part is the Jacobian (3).

\$2. The second transvectant of the two cubics $a_{2}^{3}=0$ and $b_{z}^{3}=0$ was fixed geometrically by determining the mid point of the pair of points representing it, namely $(a b)^{2} a_{z} b_{x}=0$, and fixing a pair of quadratics to which it is apolar.*

* Procetdings Edin. Math. Society, Vol. 42, Pt. I. 
This mid point may be fixed as follows.

Consider the focal points of the first and second cubics, namely $a_{0} z^{2}+2 a_{1} z+a_{2}=0=a_{z}^{2}$ and $b_{0} z^{2}+2 b_{1} z+b_{2}=0=b_{z}^{2}$. If the roots of $a_{z}^{2}=0$ are $y_{1}$ and $y_{z}$ : then determine the first polar of $y_{1}$ with respect to $b_{z}^{3}=0$, and then the first polar of $y_{3}$ with respect to the two points just found. This is the linear covariant of the quadratic

$$
\begin{gathered}
a_{z}^{2}=0 \text { and } b_{2}^{3}=0 \\
(a b)^{2} b_{z}=0=\left\{z\left(a_{3} b_{0}-2 a_{1} b_{1}+b_{2} a_{0}\right)+a_{2} b_{1}+a_{0} b_{3}-2 a_{1} b_{3}=0\right\} .
\end{gathered}
$$

Treating similarly $b_{z}^{2}=0$ and $a_{z}^{3}=0$ we get

$$
(a b)^{n} a_{z}=0=\left\{z\left(a_{2} b_{0}-2 a_{1} b_{1}+b_{2} a_{0}\right)+b_{2} a_{1}+a_{3} b_{0}-2 a_{2} b_{1}=0\right\} \text {. }
$$

The mid point of these two points is the mid point of the second transvectant $(a b)^{2} a_{z} b_{z}=0$.

Incidentally if the two points so found coincide we get an interpretation for the vanishing of the $(a b)^{3}$ or the Apolarity condition for the two cubics, $a_{z}^{3}=0 \quad b_{z}^{3}=0$.

$$
\begin{gathered}
-\frac{b_{2} a_{1}+a_{2} b_{0}-2 a_{4} b_{1}}{a_{2} b_{0}-2 a_{1} b_{1}+b_{2} a_{0}}=-\frac{a_{2} b_{1}+a_{0} b_{3}-2 a_{1} b_{2}}{a_{2} b_{0}-2 a_{1} b_{1}+b_{2} a_{0}} \\
a_{0} b_{3}-3 a_{1} b_{2}+3 a_{2} b_{1}-a_{3} b_{0}=0 .
\end{gathered}
$$

or 